\title{
Surgical Management and Outcome of Infected Pancreatic Necrosis
}

\author{
Nitin Nangare ${ }^{1}$, Ritvij Patankar ${ }^{2}$, Basavraj Nagur ${ }^{3}$ \\ ${ }^{1}$ MBBS, MS, Assistant Professor, Department of Surgery, Krishna Institute of Medical Sciences, Karad \\ ${ }^{2}$ MBBS, MS, Senior Resident, Department of Surgery, Krishna Institute of Medical Sciences, Karad \\ ${ }^{3}$ MBBS, Junior Resident, Department of Surgery, Krishna Institute of Medical Sciences, Karad
}

\begin{abstract}
Management of acute necrotizing pancreatitis has changed significantly over the past years. Early management is nonsurgically and solely supportive. Today, more patients survive the early phase of severe pancreatitis due to improvements of intensivecare medicine. Pancreatic infection is the major risk factor with regard to morbidity and mortality in the late phase of severe acute pancreatitis. Whereas early surgery and surgery for sterile necrosis can only be recommended in selected cases, pancreatic infection is a well accepted indication for surgical treatment [3]. Surgery should ideally be postponed until four weeks after the onset of symptoms as necrosis is well demarcated at that time. Four surgical techniques can be performed with comparable results regarding mortality: necrosectomy combined with (1) open packing, (2) planned staged relaparotomies with repeated lavage, (3)closed continuous lavage of the retroperitoneum, (4)closedpacking. However, closed continuous lavage of the retroperitoneum, and closed packing seem to be associated with a lower morbidity compared to the other two approaches[20,22]. Advances in radiologic imaging, new developments of interventional radiology and other minimal access interventions have revolutionized the management of many surgical conditions over the past decades. However, minimal invasive surgery and interventional therapy for infected necrosis should be limited to specific indications in patients who are critically ill and otherwise unfit for conventional surgery. Open surgical debridement is the "gold standard" for treatment of infected pancreatic and peripancreatic necrosis[20].
\end{abstract}

Keywords: Acute pancreatitis; infected necrosis; surgery; necrosectomy; morbidity; mortality.

\section{Introduction}

Acute pancreatitis is an inflammatory process that develops from damage to pancreatic acinar cells, which is caused by inappropriate activation of digestive enzymes within the cells. The mechanisms by which diverse etiological factors initiate an attack are unclear. The wide range of clinical presentations is based on the extent and severity of the inflammatory response. From a mild event that is confined to the gland to necrosis of the pancreas with attendant multiorgan dysfunction, increasing severity is associated with increased morbidity and mortality. Numerous approaches to estimate the severity of an episode have been used, from clinical estimation and biochemical markers to multivariable scoring systems. A shortcoming of these methods is their inability to assess the extent of injury to the pancreas and peripancreatic tissues. It is imperative that we identify patients with pancreatic necrosis, because morbidity and mortality rates in this subgroup are much higher. For the diagnosis of pancreatic parenchymal necrosis, intravenous contrast-enhanced CT scan is the ideal imaging method. The accepted criteria for the diagnosis of pancreatic necrosis on CT are focal or diffuse zones of non-enhanced pancreatic parenchyma, visualized during an examination with intravenous administration of contrast material $[5,8]$.

In 1985, Balthazar et al. were the first to grade severity of pancreatitis based on CT findings. Pancreatic tissue that has undergone necrosis typically encompasses the body or tail and shows decreased or no enhancement on CT and is surrounded by normally enhancing pancreatic tissue[4,7]. The focus of our study was to compare the predictive value of the Acute Physiological Assessment and Chronic Health
Evaluation (APACHE II) system with CT-visualized extent of pancreatic injury in severe necrotizing pancreatitis.

\section{Indications for Surgery}

INFECTED NECROSIS : Proven infected necrosis as well as septic complications resulting from pancreatic infection are well accepted indications for surgical treatment .The mortality rate of these patients is higher than $30 \%$, and more than $80 \%$ of fatal outcomes in acute pancreatitis are due to septic complications [1,4]. When treated non-surgically, mortality rates of up to $100 \%$ have been reported for infected necrosis associated with multiple organ failure. With surgical treatment, the mortality rate for patients with infected pancreatic necrosis was decreased to about 10 to 20 $\%$ in various specialized centers.

STERILE NECROSIS A conservative approach is accepted in sterile necrosis as long as the patient responds to therapy. However,when sterile necrosis is associated with organ failure, the role of surgery remains controversial. Some patients with sterile necrosis do not improve despite therapy in the ICU. Thus, it is generally agreed on that persistent or progressive organ complications despite maximal ICU treatment are an indication for surgery in patients with sterile necrosis (7). However, there is no established uniform definition of when a patient should be considered a nonresponder' to ICU therapy. In addition, surgery may be indicated in the rare event of rapidly progressive multiple organ failure in the first days of acute pancreatitis despite ICU therapy(fulminant acutepancreatitis'). Nevertheless, given the poor outcome with both surgical and conservative therapy and the lack of published data, the optimal therapy for this subset of patients remains unclear. 
International Journal of Science and Research (IJSR)

ISSN (Online): 2319-7064

Index Copernicus Value (2013): 6.14 | Impact Factor (2015): 6.391

\begin{tabular}{|l|l|l|}
\hline Surgical treatment & Morbidity & Mortality \\
\hline Sterile necrosis & $80 \%$ & $20 \%$ \\
\hline Infected necrosis & $30 \%$ & $70 \%$ \\
\hline
\end{tabular}

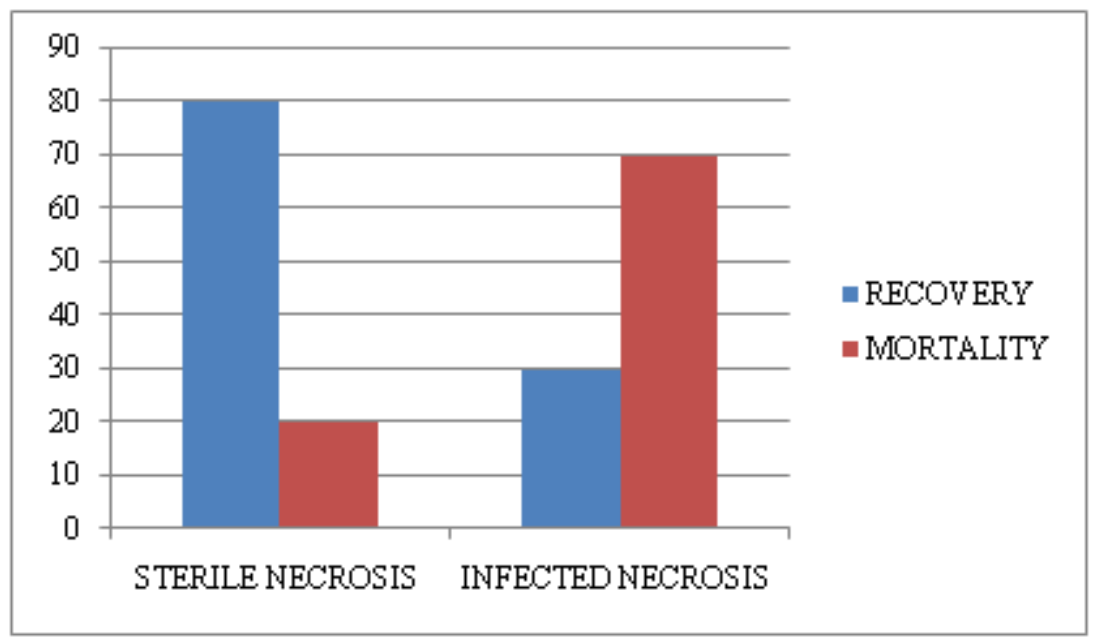

\section{Best Timepoint of Surgery}

Today, there is general agreement that surgery in severe pancreatitis should be performed as late as possible. The rationale for late surgery is the ease of identifying welldemarcated necrotic tissue from the viable parenchyma, with the effect of limiting the extent of surgery to pure debridement. This approach decreases the risk of bleeding and minimizes the surgery-related loss of vital tissue which leads to surgery-induced endocrine and exocrine pancreatic insufficiency. Mortality rates of up to $65 \%$ have been described with early surgery in severe pancreatitis, questioning the benefit of surgical intervention within the first days after onset of symptoms. In the only prospective and randomized clinical trial comparing early (within 48 to 72 hours of symptoms) versus late (at least 12 days after onset) debridement in patients with severe pancreatitis, mortality rates were $56 \%$ and $27 \%$, respectively. Although the difference did not reach statistical significance, the trial was terminated because of the evident risk of early surgery. Therefore, only in the case of proven infected necrosis or in the rare cases of severe complications such as massive bleeding or bowel perforation, early surgery must be performed.

\section{Methods :}

Data Collection. This report is a retrospective, descriptive case series. Patients who were admitted to the Krishna institute of medical hospital and research centre between June 2013 to a June 2015 with a diagnosis of acute pancreatitis were identified through the medical records system. The medical records of all patients with documented pancreatic necrosis were then reviewed. Data was collected using a standardized questionnaire. The percentage of pancreatic parenchymal necrosis was calculated by an independent review of the $\mathrm{CT}$ scans by a single radiologist (FM). Based on the extent of pancreatic necrosis on the CT scan, the patients were divided into 2 groups (group A patients, having less than $50 \%$ necrosis, and group B patients, having more than $50 \%$ necrosis) $[4,5,7,8]$. The
APACHE II score was calculated from the medical records. Patients with incomplete records or missing CT scans were excluded from the study. Cardiovascular dysfunction was defined as hypotension that required vasoactive medication; renal dysfunction as serum Creatinine levels greater than 2 $\mathrm{mg} / \mathrm{dL}$; and respiratory dysfunction as the need for mechanical ventilation or $\mathrm{PaO} 2$ levels of less than $60 \mathrm{mmHg}$. Data from reports of any cultures from surgery or fine needle aspirates (FNAs) were also collected. Infected pancreatic necrosis was defined as the presence of microorganisms in either culture. Other infections were not included in the current study.

Patients who died during the hospital stay were included in the mortality statistics.

\section{Study Design}

The study was done for 100 patients with acute pancreatitis turning necrotizing pancreatitis. Clinical outcomes were compared between groups A (minimal necrosis, i.e., <50\%) and $\mathrm{B}$ (substantial necrosis $>50 \%$ ). Also, factors were compared between survivors and nonsurvivors using univariate and multivariate analysis.

\section{Result}

The result showed that $34 \%$ of the patients went in to shock post surgery and $66 \%$ patients recovered from the shock post operatively. The patients who went in to shock did not recover and led to multi-organ failure and resulted into death. The other $66 \%$ however recovered from the shock and survived. The complications of the morbid patients included circulatory shock along with sepsis, SIRS, multiorgan failure, pleural effusion, ARDS, nephrotoxicity, hypocalcemia, etc

Thus giving a ratio of 34:66 with mortality $\mathrm{n}$ morbidity associated with infected pancreatic necrosis. 
International Journal of Science and Research (IJSR)

ISSN (Online): 2319-7064

Index Copernicus Value (2013): 6.14 | Impact Factor (2015): 6.391

\begin{tabular}{|c|c|c|}
\hline Study & Recovery & Mortality \\
\hline KIMS (100 patients) & 66 & 34 \\
\hline PANTER trial (88 patients) & 70 & 18 \\
\hline
\end{tabular}

\section{Discussion \& Conclusion}

Today, - necrosectomy and subsequent closed continuous lavage of the lesser sac" is the most commonly applied approach $(7,10)$. The differing success rates reported by groups using apparently similar approaches illustrates the difficulties in comparing these techniques (Table 1 and 2). Most techniques are associated with an average mortality between $10 \%$ and $20 \%$.However, the mortality in patients with established multiple organ failure is even higher (19). In the abscence of randomized trials, it is impossible to determine the hidden effects of factors such as referral pattern, patient selection, comorbidity of patients, presurgical percutaneous management, and indication for surgery within the literature. The high mortality in infected pancreatitic necrosis despite surgery has led to the development of several minimal invasive techniques including radiological, endoscopic, and minimal invasive surgery as alternative procedures (10). Proponents of using minimally invasive technologies in this clinical setting cite a desire to minimize the physiological insult in patients who are already critically ill $(24,25)$. However, no data exist to clearly demonstrate that minimal invasive procedures are less prone to morbidity than open surgery. Safe retroperitoneal access and necrosectomy is possible in some, but not all patients depending on size and localization of the infectious foci. Nonrandomized studies exist comparing one management technique with the other. All reports on minimal invasive surgery involve only small numbers of patients, are analysed retrospectively, and involve selected patients with an enormous variation of comorbidities and disease severity. In the absence of well-designed clinical trial, we must be cautious in the application of new technologies. Thus today, outside from clinical trials, minimal invasive surgery should be limited to specific indications and to those patients who are critically ill and otherwise unfit for conventional surgery. The role of minimal invasive surgical techniques in the treatment of infected pancreatic necrosis is outlined in detail in another article of this issue. Today, open surgical debridement is the - gld standard" for treatment of infected pancreatic and peripancreatic necrosis. - rcrosectomy and subsequent closed continuous lavage of the lesser sac" is the technique with the lowest morbidity. Consequently, it is the most commonly adopted technique to continuously remove residual pancreatic necrosis postoperatively.

\section{References}

[1] G. I. Papachristou, Prediction of severe acute pancreatitis: current knowledge and novel insights," World Journal of Gastroenterology, vol. 14, no. 41, pp. 6273-6275, 2008.

[2] G. Garcea, M. Gouda, C. Hebbes et al., Predictors of severity and survival in acute pancreatitis: validation of the efficacy of early warning scores," Pancreas, vol. 37, no. 3, pp. e54-e61, 2008.

[3] I. A. Al Mofleh, Severe acute pancreatitis: pathogenetic aspects and prognostic factors,"World
Journal of Gastroenterology, vol. 14, no. 5, pp. 675684, 2008.

[4] E. J. Balthazar, Acute pancreatitis: assessment of severity with clinical and CT evaluation," Radiology, vol. 223, no. 3, pp. 603-613, 2002.

[5] D. H. Kim and P. J. Pickhardt, -Raiblogic assessment of acute and chronic pancreatitis," Surgical Clinics of North America, vol. 87, no. 6, pp. 1341-1358, 2007.

[6] I. A. Chishty, V. Bari, S. Pasha, D. Burhan, Z. Haider, and Z. Rafique, Role of computed tomography in acute pancreatitis and its complications among age groups," Journal of the Pakistan Medical Association, vol. 55, no. 10, pp. 431-435, 2005.

[7] E. J. Balthazar, J.H. C. Ranson, D. P. Naidich, A. J.Megibow, R. Caccavale, and M. M. Cooper, Acute pancreatitis: prognostic value of CT," Radiology, vol. 156, no. 3, pp. 767-772, 1985.

[8] E. K. Paulson, K. M. Vitellas, M. T. Keogan, V. H. S. Low, and R. C. Nelson, - Aute pancreatitis complicated by gland necrosis: spectrum of findings on contrastenhanced CT," American Journal of Roentgenology, vol. 172, no. 3, pp. 609-613, 1999.

[9] Wilson P, Manji M, Neoptolemos J. Acute pancreatitis as a model of sepsis. J Antimicrob Chemother 1998; 41:51-63.

[10] Neoptolemos J, Raraty M, Finch M, Sutton R. Acute pancreatitis: the substantial human and financial costs. Gut 1998; 42:886—891.

[11] Isenmann R, Runzi M, Kron M, et al. Prophylactic antibiotic treatment in patients with predicted severe acute pancreatitis: a placebo-controlled, doubleblind trial. Gastroenterology 2004; 126:997_-1004.

[12] Uhl W, Warshaw A, Imrie C, et al. IAP guidelines for the surgical management of acute pancreatitis. Pancreatology 2002; 2:565-573.

[13] NathensAB, CurtisJR, Beale RJ, et al.Management of the critically ill patients with severe acute pancreatitis. Crit Care Med 2004; 32:2524_2536. An international consensus conference developed 23 evidence-based recommendations to address specific questions in the management of severe acute pancreatitis.

[14] Wig JD, Mettu SR, Jindal R, et al. Closed lesser sac lavage in the management of pancreatic necrosis. J Gastroenterol Hepatol 2004; 19:1010_-1015.

[15] Risse O, Auguste T, Delannoy P, et al. Percutaneous video-assisted necrosectomy for infected pancreatic necrosis. Gastroenterol Clin Biol 2004; 28:868_ 871.

[16] Connor S, Neoptolemos JP. Surgery for pancreatic necrosis: whom, when and what.' World J Gastroenterol 2004; 10:1697_-1698.

[17] King NK, Siriwardena AK. European survey of surgical strategies for the management of severe acute pancreatitis. Am J Gastroenterol 2004; 99:719_ 728.

[18] Sarr MG. IAP guidelines in acute pancreatitis: so what? [editorial]. Dig Surg 2003; 20:1—2.

[19] Nealon WH, Bawduniak J, Walser EM. Appropriate timing of cholecystectomy in patients who present with moderate to severe gallstone-associated acute pancreatitis with peripancreatic fluid collections. Ann Surg 2004; 239: 741—749.

[20] Szentkereszty Z, Kerekes L, Kotan R, et al. Non septic, surgical complications and their treatment of acute 


\section{International Journal of Science and Research (IJSR) \\ ISSN (Online): 2319-7064}

Index Copernicus Value (2013): 6.14 | Impact Factor (2015): 6.391

necrotizing pancreatitis [in Hungarian; English abstract]. Magy Seb 2004; 57:214_-218.

[21] Van MinnenLP,Besselink MG,Bosscha K,et al.Colonic involvementin acute pancreatitis: a retrospective study of 16 patients. Dig Surg 2004; 21:33_-38.

[22] Tao H-Q, Zhang J-X, Zou S-C. Clinical characteristics and management of patients with early acute severe pancreatitis: experience from a medical center in China. World J Gastroenterol 2004; 10:919—921. This paper identifies a large proportion of patients with ACS among 297 patients with severe pancreatitis.

[23] Labler L, Keel M, Trentz O. New application of V.A.C. (vacuum assisted closure) in the abdominal cavity in case of open abdomen therapy [in German; English abstract]. Zentralbl Chir 2004; 129:S14_S19.

[24] Raraty MG, Halloran CM, Dodd S, et al. Minimal access retroperitoneal pancreatic necrosectomy: improvement in morbidity and mortality with a less invesive approach. Ann Surg 2010; 251 : 787-93. 